\title{
Nonlinear filtering in target tracking using cooperative mobile sensors *
}

\author{
Jiangping $\mathrm{Hu}^{\mathrm{a}}$, Xiaoming $\mathrm{Hu}^{\mathrm{a}, *}$ \\ ${ }^{a}$ Optimization and Systems Theory and ACCESS Linnaeus Center, \\ Royal Institute of Technology, SE-100 44 Stockholm, Sweden
}

\begin{abstract}
Collaborative signal processing and sensor deployment have been among the most important research tasks in target tracking using networked sensors. In this paper, the mathematical model is formulated for single target tracking using mobile nonlinear scalar range sensors. Then a sensor deployment strategy is proposed for the mobile sensors and a nonlinear convergent filter is built to estimate the trajectory of the target.
\end{abstract}

Key words: Nonlinear filtering, equilateral-triangle deployment, formation control, mobile sensors.

\section{Introduction}

Sensor networks have the potential ability to monitor and instrument the real world (Arge, \& Clare (2000); Kumar, Zhao, \& Shepherd (2002); Zhao, Shin, \& Reich (2002); Brooks, Ramanathan, \& Saveed (2003)). Among many challenges of sensor networks, tracking target by using collaborative information processing and optimal networking has been an important problem in both theory and application.

In this paper, we consider single target tracking by using a group of mobile distributed nonlinear sensors such as range and direction sensors in terms of sensor deployment. If the measurement of the target for each sensor is nonlinear and noisy, nonlinear filtering theory has to be applied to estimate the state of the target. In most cases, the target's dynamics is partially or even completely unknown, which makes the modeling and estimation problem for target tracking more complicated. Subsequently the target's dynamics will be described by a linear Gaussian model and the distributed sensors' measurements for the target will be nonlinear and noisy. Our aim is to propose a convergent nonlinear filter to estimate the trajectory of the target based on the measurements from the scalar range sensors. Considering

* This paper was not presented at any IFAC meeting.

* Corresponding author: Xiaoming Hu. Tel. +46-8-790 7180. Fax +46-8-22 5320 .

Email addresses: hjp_lzu@163.com (Jiangping $\mathrm{Hu}$ ), hu@math.kth.se (Xiaoming $\mathrm{Hu}$ ). the sensors' tracking qualities, sensor deployment has to be dealt with by adopting formation control techniques. This idea has also been adopted in, for example, Martinez, \& Bullo (2006), where the authors integrated motion coordination strategies and extended Kalman filter to improve the tracking performance of the sensor network. In Cheng, Ghosh, \& Hu (2006) collaborative localization algorithms are proposed to reconstruct the position of the target in a noise-free environment.

In many real applications, target tracking has to be dealt with in the presence of disturbed target dynamics and nonlinear noisy measurements. The estimation in nonlinear systems has been an extremely important problem (Julier, \& Uhlmann (2004)). Though there are now many nonlinear filtering algorithms, such as point-based unscented filters, density-based particle filters, the extended Kalman filter (EKF) has been a popular filter for nonlinear systems, in particular for target tracking problems (Martinez, \& Bullo (2006); Sugathadasa,Martin, \& Dayawansa (2000)). However, little work has been performed to analyze the stability and convergence of the filter, see Reif,Gunther,Yaz, \& Unbehauen (2000) and references therein. It is well known that the upper (lower) bound of the solution of the Riccati equation associated with EKF depends on the uniform controllability (uniform detectability) of the considered nonlinear systems (see Baras,Bensoussan, \& James (1988), Theorem 7). However, with the model used in this paper, even though EKF has a comparative accuracy, it is difficult to show the convergence of EKF since the uniform complete con- 
trollability cannot be assumed. In addition, the Riccati differential equations associated with the error covariance are very difficult to integrate numerically. Then an important problem arises: can we design a convergent nonlinear filter to track the target with unknown dynamics?

The contribution of this paper is twofold: one is the proposal of a convergent nonlinear filter and the other is for appropriate deployment of the sensors. Sensor deployment concerns the connectivity and coverage of the network, which plays a key role in energy conservation and monitoring quality (Wang.Xing, et al. (2003); Kumar, Lai, \& Balogh (2004)) and, at the same time, assures the feasibility of our proposed filter. A fundamental problem, i.e., $k$-coverage problem, facing sensor deployment for target tracking is how to deploy the sensors so that every point in the target region will be monitored by at least $k$ sensors. For target tracking with mobile sensors, since the deployment algorithm will be integrated with the estimation process, $k$-coverage problems become more difficult to solve. In this paper, inspired by a virtual vehicle approach in Egerstedt.Hu.\& Stotsky (2001), neighbor-based formation control will be proposed to achieve an equilateraltriangle deployment and solve the 3 -coverage problem for mobile sensors.

The rest of the paper is organized as follows. In Section 2, we discuss the sensor deployment and formulate the mathematical model in the target tracking problem. In Section 3, on the basis of equilateral-triangle deployment for mobile sensors, the design and convergence of a nonlinear filter will be given to track the target. In Section 4 , the 3 -coverage problem will be solved for mobile sensors by designing a decentralized control to achieve equilateral-triangle formation.

Throughout this paper, we will use the following notations: $A$ is a real matrix, $A^{T}$ denotes its matrix transpose, $\operatorname{tr}(A)$ is the trace of $A, \lambda_{i}(A)$ is an eigenvalue of $A,\|A\|_{2}$ is the spectral norm; for vector $x,\|x\|_{2}$ is Euclidean norm and $\|x\|_{\infty}$ is maximum norm; $<\cdot>$ denotes the inner product in Euclidean space; $\otimes$ denotes the Kronecker product; $E[\cdot]$ is the expectation operator; $\operatorname{col}(\cdot)$ denotes the concatenation; Dirac $(\cdot)$ is the Dirac delta operator.

\section{Problem formulation}

In this section, we will formulate the target tracking problem on sensor deployment strategy and convergent nonlinear filter design.

Consider target tracking with $N$ mobile sensors. Suppose that each sensor is modeled as two disks with radii $r$ and $r_{c}$, which indicate the sensing range and the communication range, respectively. In order to improve the tar- get tracking quality, having the target sensed by at least $k$ (no less than 3) sensors in a plane is critical. Meanwhile, the sensors should be kept connected to transmit and receive data successfully. It has been shown in Wang.Xing, et al. (2003) that if $r_{c} \geq 2 r, k$-coverage implies connectivity of the network. For the case the target region is constrained in a space of dimension two, the problem of designing a target tracking filter will be resolved in Section 3 and, the problem of how to make the region be 3 -covered by mobile sensors in Section 4 .

Let the kinematic equation of sensor $i$ be described by a nonlinear system

$$
\dot{s}_{i}(t)=f_{i}\left(s_{i}(t), u_{s, i}(t)\right), i=1, \cdots, N,
$$

where $s_{i}(t) \in \mathbb{R}^{n}$ is the position of sensor $i$ and $u_{s, i}(t)$ is its input. The measurement of the target for each scalar sensor is nonlinear and noisy and can be expressed as

$$
y_{i}(t)=\phi_{i}\left(p(t), s_{i}(t)\right)+\omega_{i}(t), i=1, \cdots, N,
$$

where $\phi_{i} \in \mathbb{R}$ is continuously differentiable with respect to $p$ and $\omega_{i}$ are independent Gaussian white noises with covariance matrices $E\left[\omega_{i}\left(t_{1}\right) \omega_{i}^{T}\left(t_{2}\right)\right]=\Omega_{i} \operatorname{Dirac}\left(t_{1}-t_{2}\right)$. The variable $p(t)\left(\in \mathbb{R}^{n_{1}}\right)$ denotes the state of the target. When $p(t)$ represents the position or orientation angle of the target, the sensors will be called range sensors. Another kind of sensors is called velocity sensor if $p$ contains the velocity vector of the target.

Now suppose that the state $p(t)$ of the target evolves in a continuous-time linear system with partially unknown input:

$$
\left\{\begin{array}{l}
\dot{p}(t)=A p(t)+B u(t), \\
p\left(t_{0}\right)=p_{0},
\end{array}\right.
$$

where $u \in \mathbb{R}^{n_{2}}\left(n_{2} \leq n_{1}\right)$ is the partially unknown input, the initial state $p\left(t_{0}\right)$ is also an unknown constant vector and $A, B$ are constant matrices. The unknown input is generated by a linear exogenous system

$$
\left\{\begin{array}{l}
\dot{\zeta}(t)=\Gamma \zeta(t)+F \nu(t), \\
u(t)=D \zeta(t)
\end{array}\right.
$$

where $\zeta \in \mathbb{R}^{m}, \nu \in \mathbb{R}^{n_{3}}, \Gamma, F, D$ are known matrices and the system disturbance $\nu$ is a zero-mean Gaussian white noise with $E\left[\nu(t) \nu^{T}(s)\right]=\Psi \operatorname{Dirac}(t-s)$.

Then the target's dynamics (3) and the kinematics (1) and the measurements (2) of $N$ sensors compose an extended system

$$
\begin{gathered}
\left(\begin{array}{l}
\dot{p} \\
\dot{\zeta}
\end{array}\right)=\bar{A}\left(\begin{array}{l}
p \\
\zeta
\end{array}\right)+\bar{B} \nu, \\
\dot{s}=f\left(s, u_{s}\right),
\end{gathered}
$$




$$
y=\phi(p, s)+\omega
$$

where $y=\operatorname{col}\left(y_{1}, \cdots, y_{N}\right), s=\operatorname{col}\left(s_{1}, \cdots, s_{N}\right), u_{s}=$ $\operatorname{col}\left(u_{s, 1}, \cdots, u_{s, N}\right), f=\operatorname{col}\left(f_{1}, \cdots, f_{N}\right), \omega=\operatorname{col}\left(\omega_{1}\right.$, $\left.\cdots, \omega_{N}\right)$ and

$$
\bar{A}=\left(\begin{array}{cc}
A & B D \\
0 & \Gamma
\end{array}\right), \bar{B}=\left(\begin{array}{l}
0 \\
F
\end{array}\right) .
$$

Remark 1 Here we cannot assume that system $(\bar{A}, \bar{B})$ is (uniformly) controllable. Thus, the boundedness of the solution of the Riccati equation associated with EKF corresponding to system (5), (7) can not be guaranteed.

Let $C(p, s)$ be the Jacobian matrix of $\phi(p, s)$ with respect to $p$ and $\bar{C}=\left[C(p, s), 0_{N \times m}\right]$. If there is no confusion, $C(p, s)$ will be abbreviated as $C$ in the sequel. When the observability of time-varying system with $C$ or $\bar{C}$ is mentioned, $p$ and $s$ are regarded as constants. In order to investigate the observability of system $(\bar{A}, \bar{C})$, we make an assumption as follows:

Assumption 2 The pairs $(A, C)$ and $(\Gamma, D)$ are observable and no eigenvalue of $\Gamma$ is a transmission zero of system $(A, B, C)$.

The following result can be checked by Hautus test and the proof is omitted here (the detail can be found in Hu. \& Hu (2008)).

Lemma 3 Under Assumption 2, the matrix pair $(\bar{A}, \bar{C})$ is observable.

Remark 4 For system $(\bar{A}, \bar{B}, \bar{C})$ in this paper, we will show in Section 3 that, in fact, $(\bar{A}, \bar{C})$ will be also uniformly detectable by finding a gain matrix $H(p, s)$ such that $\bar{A}+H \bar{C}$ will have negative real-part eigenvalues for arbitrary time-varying variables $p$ and $s$.

In terms of Lemma 3, a nonlinear observer-based filter can be proposed for systems (5) and (7):

$$
\left\{\begin{array}{l}
\dot{\hat{p}}=A \hat{p}+B D \hat{\zeta}+H_{p}(y-\phi(\hat{p}, s)), \\
\dot{\hat{\zeta}}=\Gamma \hat{\zeta}+H_{\zeta}(y-\phi(\hat{p}, s)) .
\end{array}\right.
$$

In this paper, we limit ourselves to nonlinear observers that satisfy the following constraints: 1) $H_{p}, H_{\zeta}$ are gain matrices to be designed such that $H_{p} C(\hat{p}, s)$ and $H_{\zeta} C(\hat{p}, s)$ are constant matrices; 2$)$ the observer is an asymptotic observer for the given system in the absence of system disturbance $\nu$ and measurement noise $\omega$. The motivation for the second constraint is that the observer should be an possible asymptotic observer even when some, or all, components of the system state or the measurements are noise free.
The nonlinear function $\phi(p, s)$ can be expanded up to first order via

$$
\phi(p, s)=\phi(\hat{p}, s)+C(\hat{p}, s)(p-\hat{p})+\Delta(p, \hat{p}, s),
$$

where $\Delta(p, \hat{p}, s)$ is the remaining nonlinear term. The estimation errors are defined by $e_{p}=p-\hat{p}, e_{\zeta}=\zeta-\hat{\zeta}$. Then we can have the nonlinear error dynamics

$$
\left\{\begin{array}{l}
\dot{e_{p}}=\left(A-H_{p} C(\hat{p}, s)\right) e_{p}+B D e_{\zeta}-H_{p} \Delta(p, \hat{p}, s)-H_{p} \omega, \\
\dot{e_{\zeta}}=\Gamma e_{\zeta}-H_{\zeta} C(\hat{p}, s) e_{p}-H_{\zeta} \Delta(p, \hat{p}, s)-H_{\zeta} \omega+F \nu .
\end{array}\right.
$$

To examine the error dynamics (10), consider a general Ito stochastic differential equation

$$
d e(t)=g(e(t)) d t+b(t) d \tilde{\omega}(t)
$$

where $\tilde{\omega}(t)$ is a standard Brown motion.

Then a differential generator associated with $e(t)$ is defined as follows (Zakai M. (1967)):

$$
\mathcal{L}(\cdot)=\frac{\partial(\cdot)}{\partial e} g(e(t))+\frac{1}{2} \operatorname{tr}\left(b(t) b^{T}(t) \operatorname{Hess}(\cdot)\right)
$$

where Hess $(\cdot)$ denotes the Hessian matrix.

Now a key lemma to show the stochastic boundedness or stability is given as follows Reif.Gunther.Yaz.\& Unbehauen (2000); Zakai M. (1967)):

Lemma 5 If there is a stochastic process $V(e(t))$ and positive numbers $\lambda_{1}, \lambda_{2}, \lambda_{3}, \epsilon$ such that

$$
\lambda_{1}\|e(t)\|^{2} \leq V(e(t)) \leq \lambda_{2}\|e(t)\|^{2}
$$

and

$$
\mathcal{L} V(e(t)) \leq-\lambda_{3} V(e(t))+\epsilon
$$

are fulfilled, then the stochastic process $e(t)$ is exponentially bounded in mean square, i.e.

$$
E\left[\|e(t)\|^{2}\right] \leq \frac{\lambda_{2}}{\lambda_{1}}\|e(0)\| e^{-\lambda_{3} t}+\frac{\epsilon}{\lambda_{1} \lambda_{3}}
$$

for every $t \geq 0$.

In the subsequent sections, we will firstly give a construction of nonlinear filter (8) and analyze its stability. Secondly we will discuss a deployment for mobile sensors in order to ensure the existence of nonlinear filter (8) and improve the tracking quality as well.

\section{$3 \quad$ Filter design and analysis}

In this section, a nonlinear filter will be built to track the target by using mobile range sensors. Then the stochastic stability of the filter will be analyzed. 


\subsection{Target tracking filter design}

Suppose that the target dynamics (3) is expressed as follows:

$$
\left\{\begin{array}{l}
\dot{x}=v, \\
\dot{v}=u
\end{array}\right.
$$

where $x, v, u \in \mathbb{R}^{n}$ denote the position, velocity and acceleration of the target, $p=\operatorname{col}(x, v)$,

$$
A=\left(\begin{array}{cc}
0 & I_{n} \\
0 & 0
\end{array}\right), B=\left(\begin{array}{c}
0 \\
I_{n}
\end{array}\right)
$$

Additionally, the acceleration $u$ is given by the exogenous system (4).

In the rest of the paper, we will consider only scalar range sensors whose measurements of target are given by

$$
y_{i}(t)=\phi_{i}\left(x(t), s_{i}(t)\right)+\omega_{i}(t) \in \mathbb{R}, i=1, \cdots, N
$$

or,

$$
y=\phi(x, s)+\omega \in \mathbb{R}^{N}
$$

To reconstruct the state of the target, an assumption on the rank of Jacobian matrix $C(x, s)$ is made:

Assumption 6 For continuous differentiable function $\phi(x, s)$, there exists a number $N$ such that the Jacobian matrix $C(x, s)=\frac{\partial \phi}{\partial x}(x, s)$ is column full rank.

The assumption suggests that when enough number of sensors are placed in a finite dimensional state space, we can always find that the gradients $\frac{\partial \phi_{i}(x, s)}{\partial x}$ are linearly independent and then the reconstruction of the states of the target will be possible. As $N$ turns larger, the assumption on Jacobian rank becomes satisfied more easily. For example, if we take the measurement function as

$$
\phi(x, s)=\operatorname{col}\left(\left\|x-s_{1}\right\|, \cdots,\left\|x-s_{N}\right\|\right) .
$$

It follows that the Jacobian matrix is

$$
\operatorname{col}\left(\frac{\left(x-s_{1}\right)^{T}}{\left\|x-s_{1}\right\|}, \cdots, \frac{\left(x-s_{N}\right)^{T}}{\left\|x-s_{N}\right\|}\right) .
$$

When three or more sensors are placed in the plane, it is easy to find non-collinear positions relative to the target for the sensors. This is just the reason why we should consider the 3-coverage problem in mobile sensor network in next section.

In what follows, for the sake of constructing a filter for the extended system (16), (4) and (18), we investigate the structure of system (4). From Assumption 2, $(\Gamma, D)$ is observable, then there exists a matrix $G \in \mathbb{R}^{m \times n}$ such that $\Gamma-G D$ is a Hurwitz matrix. Then, for an arbitrary positive definite matrix $Q_{\zeta} \in \mathbb{R}^{m \times m}$, there is a symmetric positive definite matrix $P_{\zeta} \in \mathbb{R}^{m \times m}$ such that

$$
P_{\zeta}(\Gamma-G D)+(\Gamma-G D)^{T} P_{\zeta}=-Q_{\zeta}
$$

The following assumption is given to guarantee the convergence of the proposed nonlinear filter (8) associated with the extended system (16), (4) and (18).

Assumption 7 For gain matrix $G$ in equation (20), we have the following two relations:

(1) $P_{\zeta}(\Gamma-G D) G+\varpi D^{T} P_{1}=0$,

(2) $P_{1}\left(D G-\frac{1}{\varpi} I_{n}\right)+\left(D G-\frac{1}{\varpi} I_{n}\right)^{T} P_{1}=-Q_{1}$,

where $\varpi>1$ and $Q_{1}, P_{1}$ are $n$-dimensional symmetric positive definite matrices.

Now a nonlinear filter is proposed as follows:

$$
\left(\begin{array}{c}
\dot{\hat{p}} \\
\dot{\hat{\zeta}}
\end{array}\right)=\left(\begin{array}{cc}
A & B D \\
0 & \Gamma
\end{array}\right)\left(\begin{array}{l}
\hat{p} \\
\hat{\zeta}
\end{array}\right)+H(\hat{x}, s)(y-\phi(\hat{x}, s))
$$

where $\hat{p}=\left(\hat{x}^{T}, \hat{v}^{T}\right)^{T}$, and the gain matrix $H(\hat{x}, s)=$ $M J_{\hat{x}}^{-1} C^{T}(\hat{x}, s)$,

$$
\begin{aligned}
M & =\left(\begin{array}{c}
(\alpha-1) I_{n}-\frac{1}{\varpi-1} P_{1}^{-1} G^{T} D^{T} P_{1} \\
\frac{\alpha}{\varpi} I_{n}-\frac{1}{\varpi-1} P_{1}^{-1} G^{T} D^{T} P_{1} \\
G\left(\frac{\alpha}{\varpi} I_{n}-\frac{1}{\varpi-1} P_{1}^{-1} G^{T} D^{T} P_{1}\right)-P_{\zeta}^{-1} D^{T} P_{1}
\end{array}\right), \\
J_{\hat{x}} & =\frac{\partial^{T} \phi}{\partial x}(\hat{x}, s) \frac{\partial \phi}{\partial x}(\hat{x}, s)=\sum_{i=1}^{N} \frac{\partial \phi_{i}}{\partial x}(\hat{x}, s) \frac{\partial^{T} \phi_{i}}{\partial x}(\hat{x}, s), \\
\alpha>\frac{\varpi}{\varpi-1} &
\end{aligned}
$$

With Assumption 6, the inverse of $J_{\hat{x}}$ is well defined.

\subsection{Stability analysis}

Now we turn to the stability analysis of filter (21).

Define $e_{p}=p-\hat{p}, e_{\zeta}=\zeta-\hat{\zeta}$, then the nonlinear error dynamics (10) can be rewritten in a compact form:

$$
\dot{e}=\hat{A} e-H(\hat{x}, s) \Delta(x, \hat{x}, s)+\bar{B} \nu-H \omega,
$$

where

$$
e=\left(\begin{array}{c}
e_{p} \\
e_{\zeta}
\end{array}\right), \hat{A}=\bar{A}-H(\hat{x}, s) \bar{C}(\hat{x}, s), \bar{B}=\left(\begin{array}{c}
0_{2 n \times m} \\
F
\end{array}\right)
$$


and $\Delta(x, \hat{x}, s)$ satisfies

$$
\phi(x, s)=\phi(\hat{x}, s)+C(\hat{x}, s)(x-\hat{x})+\Delta(x, \hat{x}, s) .
$$

Assumption 8 There are positive numbers $\delta, \chi$ such that the remaining term $\Delta(x, \hat{x}, s)$ is bounded by

$$
\|\Delta(x, \hat{x}, s)\| \leq \chi\|x-\hat{x}\|^{2}
$$

for $\|x-\hat{x}\| \leq \delta$

Assumption 9 There is a positive number $\gamma$ such that the smallest eigenvalue of matrix $J_{\hat{x}}$ is satisfied for all $t \geq 0$ :

$$
\lambda_{\min }\left(J_{\hat{x}}\right) \geq \gamma
$$

Remark 10 If we can resolve the 3 -coverage problem in the mobile sensor network, this assumption will be fulfilled since $J_{\hat{x}}$ is bounded positive definite when $N$ sensors are deployed in the interested area (compact subset of Euclidean space) with equilateral-triangle formation, which will be considered in Section 4.

Definition 11 In equation (22), if there exist positive numbers $a_{1}, a_{2}, a_{3}$ such that

$$
E\left[\|e(t)\|^{2}\right] \leq a_{1}\|e(0)\| e^{-a_{2} t}+a_{3}
$$

holds for every $t \geq 0$, we say that the nonlinear filter (21) can track the target.

Now a main result will be presented as follows:

Theorem 12 Consider a target with disturbed dynamics (16), (4) and $N$ mobile sensors with nonlinear measurements (18). Under Assumptions 2, 7, 8 and 9, the filter defined by (21) can track the target.

Proof: Firstly, for system $(22)$, we will show that $(\bar{A}, \bar{C})$ with given gain matrix $H(\hat{x}, s)$ will be stabilizable under Assumption 2. A variable transform is defined as follows:

$$
\tilde{e}=T e,
$$

where

$$
T=\left(\begin{array}{ccc}
I_{n} & 0 & 0 \\
0 & I_{n} & 0 \\
0 & -G & I_{m}
\end{array}\right)
$$

Then the error dynamics (22) is transformed to

$$
\dot{\tilde{e}}=\tilde{A} \tilde{e}-T H \Delta(x, \hat{x}, s)+T \bar{B} \nu-T H \omega,
$$

where $\tilde{A}=T \hat{A} T^{-1}$.
Define $V(\tilde{e})=\tilde{e}^{T} P \tilde{e}$, where $P$ is a symmetric matrix such that

$$
P=\left(\begin{array}{ccc}
P_{1} & -P_{1} & 0 \\
-P_{1} & \varpi P_{1} & 0 \\
0 & 0 & P_{\zeta}
\end{array}\right)(\varpi>1)
$$

and $P_{1}, P_{\zeta}$ and $\varpi$ satisfy Assumption 7 . It is easy to see that $P$ is positive definite by Schur Complement Formula. Obviously, $V(\tilde{e})$ is bounded by

$$
\lambda_{\min }(P) \tilde{e}^{T} \tilde{e} \leq V(\tilde{e}) \leq \lambda_{\max }(P) \tilde{e}^{T} \tilde{e}
$$

where $\lambda_{\min }(P)$ and $\lambda_{\max }(P)$ are the smallest and largest eigenvalue of $P$, respectively.

For the given stochastic process $V(\tilde{e})$, the differential generator is given by

$$
\begin{aligned}
\mathcal{L} V(\tilde{e})= & \tilde{e}^{T}\left(P \tilde{A}+\tilde{A}^{T} P\right) \tilde{e}-2 \tilde{e}^{T} P T H \Delta(x, \hat{x}, s) \\
& +\operatorname{tr}\left(P T \bar{B} \bar{B}^{T} T^{T}\right)+\operatorname{tr}\left(P T H H^{T} T^{T}\right) .
\end{aligned}
$$

Under Assumption 7, it is not difficult to obtain that

$\tilde{A}=\left(\begin{array}{ccc}\frac{1}{\varpi-1} P_{1}^{-1} G^{T} D^{T} P_{1}-(\alpha-1) I_{n} & I_{n} & 0 \\ \frac{1}{\varpi-1} P_{1}^{-1} G^{T} D^{T} P_{1}-\frac{\alpha}{\varpi} I_{n} & D G & D \\ P_{\zeta}^{-1} D^{T} P_{1} & (\Gamma-G D) G \Gamma-G D\end{array}\right)$,

thus we have

$Q=:-\left(P \tilde{A}+\tilde{A}^{T} P\right)=\operatorname{Diag}\left\{2\left(\alpha-\frac{\alpha}{\varpi}-1\right) P_{1}, \varpi Q_{1}, Q_{\zeta}\right\}$,

which is a positive definite matrix due to Assumption 7 and $\alpha>\frac{\varpi}{\varpi-1}$. Thus, the matrices $\tilde{A}$ and $\hat{A}$ will be Hurwitz stable.

Let $\lambda_{\min }(Q)$ be the smallest eigenvalue of $Q$. By Assumptions 8,9 , then the generator is

$$
\begin{aligned}
\mathcal{L} V(\tilde{e}) \leq & -\left(\lambda_{\min }(Q)-2 \chi \lambda_{\max }(P)\|T\|_{2}\|H\|_{2}\|\tilde{e}\|\right) \tilde{e}^{T} \tilde{e} \\
& +\lambda_{\max }(P)\|T\|_{2}^{2}\left(\operatorname{tr}\left(F F^{T}\right)+\operatorname{tr}\left(H H^{T}\right)\right) .
\end{aligned}
$$

Since $\|H\|_{2}^{2} \leq \operatorname{tr}\left(H H^{T}\right) \leq\|M\|_{2}^{2} \operatorname{tr}\left(J_{\hat{x}}^{-1}\right) \leq n \frac{\|M\|_{2}^{2}}{\gamma}$, we have

$$
\begin{aligned}
\mathcal{L} V(\tilde{e}) \leq & -\left(\lambda_{\min }(Q)-2 \chi \lambda_{\max }(P) \sqrt{\frac{n}{\gamma}}\|M\|_{2}\|T\|_{2}\|\tilde{e}\|\right) \tilde{e}^{T} \tilde{e} \\
& +\lambda_{\max }(P)\|T\|_{2}^{2}\left(\operatorname{tr}\left(F F^{T}\right)+n \frac{\|M\|_{2}^{2}}{\gamma}\right) .
\end{aligned}
$$


Defining

$$
\begin{gathered}
\tilde{\delta}=\min \left(\|T\|_{2} \delta, \frac{\lambda_{\min }(Q)}{4 \chi \lambda_{\max }(P) \sqrt{\frac{n}{\gamma}}\|T\|_{2}\|M\|_{2}}\right), \\
\epsilon=\lambda_{\max }(P)\|T\|_{2}^{2}\left(\operatorname{tr}\left(F F^{T}\right)+n \frac{\|M\|_{2}^{2}}{\gamma}\right),
\end{gathered}
$$

and using (29) one obtains $\mathcal{L} V(\tilde{e}) \leq-\eta V(\tilde{e})+\epsilon$ with $\eta=\frac{\lambda_{\min }(Q)}{2 \lambda_{\max }(P)}$ for $\|\tilde{e}\| \leq \tilde{\delta}$. According to Lemma 5 ,

$$
E\left[\|\tilde{e}(t)\|^{2}\right] \leq \frac{\lambda_{\max }(P)}{\lambda_{\min }(P)}\|\tilde{e}(0)\| e^{-\eta t}+\frac{\epsilon}{\lambda_{\min }(P) \eta},
$$

by Definition 11 and the transformation (26), the conclusion follows.

\section{Motion coordination of sensors}

In this section, we discuss conditions for deploying the mobile sensors that guarantee Assumption 6 and Assumption 9, which play an essential role in the proposed filter design. We will design neighbor-based control so the mobile sensors move in equilateral-triangle formations.

Suppose that the interconnection topology of the sensor network is a tree. For range sensors, the tree interconnection topology means that sensor $i$ can measure the position (i.e. distance and bearing angle with respect to sensor $i$ ) of sensor $i-1$ for $i=1, \cdots, N$. Additionally, suppose that all sensor can get the states of the estimated target, which can be regarded as a virtual sensor labeled 0.

A unicycle model is used to describe the kinematics of sensor $i$ for $i=1, \cdots, N$, i.e.

$$
\begin{aligned}
\dot{x}_{s, i} & =v_{s, i} \cos \varphi_{i}, \\
\dot{y}_{s, i} & =v_{s, i} \sin \varphi_{i}, \\
\dot{\varphi}_{i} & =\mathrm{w}_{\mathrm{i}},
\end{aligned}
$$

where $s_{i}=\left(x_{s, i}, y_{s, i}\right)^{T} \in \mathbb{R}^{2}$ is the position, $v_{s, i}$ is the translational velocity, $\varphi_{i}$ is the orientation angle and $\mathrm{w}_{i}$ is the angular velocity. Here, the control to be designed is $v_{s, i}$ and $\mathrm{w}_{i}$.

Let $d_{i}$ denote the actual distance between sensor $i$ and $i-1$ (while $d_{0, i}>0$ is the corresponding desired distance), let $\beta_{i}$ be the actual bearing angle from the orientation of sensor $i$ to edge $\vec{s}_{i} s_{i-1}$ and $\beta_{0, i}$ the corresponding desired bearing angle (see Fig. 1). Here, $\beta_{i}$ and $\beta_{0, i}$ are constrained in the radian interval $[-\pi,-\pi / 2) \bigcup(-\pi / 2, \pi / 2) \bigcup(\pi / 2, \pi]$. Note that the desired formation is defined by $d_{i}=d_{0, i}$ and $\beta_{i}=\beta_{0, i}$ for $i=1, \cdots, N$. Finally, define $\theta_{i}=\varphi_{i}-\varphi_{i-1}$.

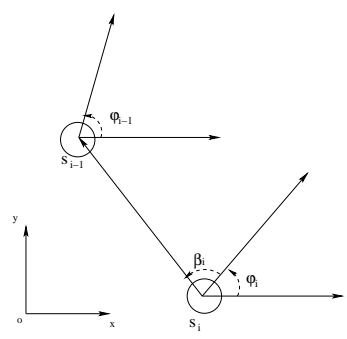

Fig. 1. Notations of angles and distances

Then we can rewrite the unicycle model (33) as

$$
\begin{aligned}
\dot{d}_{i} & =-v_{s, i} \cos \beta_{i}-v_{s, i-1} \cos \left(\pi-\theta_{i}-\beta_{i}\right), \\
\dot{\theta}_{i} & =\mathrm{w}_{\mathrm{i}}-\mathrm{w}_{\mathrm{i}-1}, \\
\dot{\beta}_{i} & =-\mathrm{w}_{i}+\frac{v_{s, i}}{d_{i}} \sin \beta_{i}-\frac{v_{s, i-1}}{d_{i}} \sin \left(\pi-\theta_{i}-\beta_{i}\right) .
\end{aligned}
$$

Inspired by the path-following control presented in Egerstedt,Hu, \& Stotsky (2001), a virtual sensor approach will be used. A reference point $\left(x_{s, i}^{0}, y_{s, i}^{0}\right)$ is chosen on $s_{i}$ 's axis of orientation at a distance $d_{0, i}$ with bearing angle $\beta_{0, i}$. Then we have

$$
\begin{gathered}
x_{s, i}^{0}=x_{s, i}+d_{0, \mathrm{i}} \cos \left(\varphi_{i}+\beta_{0, \mathrm{i}}\right), \\
y_{s, i}^{0}=y_{s, i}+d_{0, \mathrm{i}} \sin \left(\varphi_{i}+\beta_{0, \mathrm{i}}\right) .
\end{gathered}
$$

Derivation of equation (35) in combination with unicycle model (33) gives the following relationship:

$$
\left(\begin{array}{c}
v_{\mathrm{s}, \mathrm{i}} \\
\mathrm{w}_{\mathrm{i}}
\end{array}\right)=\left(\begin{array}{cc}
\frac{\cos \left(\varphi_{i}+\beta_{0, \mathrm{i}}\right)}{\cos \beta_{0, \mathrm{i}}} & \frac{\sin \left(\varphi_{i}+\beta_{0, \mathrm{i}}\right)}{\cos \beta_{0, \mathrm{i}}} \\
-\frac{\sin \varphi_{i}}{d_{0, \mathrm{i}} \cos \beta_{0, \mathrm{i}}} & \frac{\cos \varphi_{i}}{d_{0, \mathrm{i}} \cos \beta_{0, \mathrm{i}}}
\end{array}\right)\left(\begin{array}{c}
\dot{x}_{s, i}^{0} \\
\dot{y}_{s, i}^{0}
\end{array}\right)-\left(\begin{array}{c}
0 \\
\dot{\beta}_{0, \mathrm{i}}
\end{array}\right) .
$$

On the one hand, the initial values of $\beta_{0, i}$ can be taken in $\beta_{0, i} \in[-\pi,-\pi / 2) \bigcup(-\pi / 2, \pi / 2) \bigcup(\pi / 2, \pi]$ in order to achieve equilateral-triangle formation with tree interconnection topology. However, once the target changes its orientation very fast, it is both necessary and efficient to decrease response formation time for target tracking in practice. Hence, we need to regulate the desired bearing angles timely.

An appropriate choice for the dynamics of the desired bearing angle $\beta_{0, i}$ is given by:

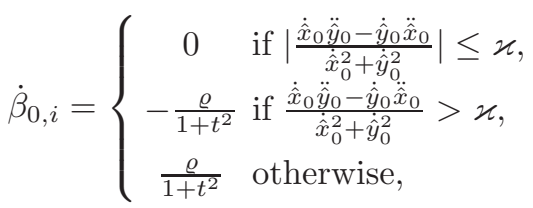

for some threshold $\varkappa>0$ and $0 \leq \varrho \leq 1$. 
On the other hand, if $\dot{x}_{s, i}^{0}$ and $\dot{y}_{s, i}^{0}$ are chosen as

$$
\begin{aligned}
\dot{x}_{s, i}^{0} & =-k_{i}\left(x_{s, i}^{0}-x_{s, i-1}\right)+\dot{x}_{s, i-1}, \\
\dot{y}_{s, i}^{0} & =-k_{i}\left(y_{s, i}^{0}-y_{s, i-1}\right)+\dot{y}_{s, i-1},
\end{aligned}
$$

for positive number $k_{i}$, the virtual sensor of sensor $i$ will be driven to sensor $i-1$. Note that when $i=1$, $\left(x_{s, i-1}, y_{s, i-1}\right)^{T}=\left(\hat{x}_{0}, \hat{y}_{0}\right)^{T}$ will be the position of virtual sensor 0 .

However, for range sensor $i(\geq 2)$, it is difficult to obtain the information of the velocity of its neighbor, i. e. sensor $i-1$, so we need an assumption on the velocities of all mobile sensors:

Assumption 13 For both sensor $i(1 \leq i \leq N)$ and the target, the velocities are bounded, i.e., $\sqrt{\dot{x}_{s, i}^{2}+\dot{y}_{s, i}^{2}} \leq k_{s}$, and $\sqrt{\dot{x}_{0}^{2}+\dot{y}_{0}^{2}} \leq k_{v}$, for some positive $k_{s}$ and $k_{v}$.

If the estimation error of the target's velocity is small enough, from Assumption 13, the virtual sensor $i(1 \leq$ $i \leq N)$ will still approach to sensor $i-1$ when the gains $k_{i}$ are taken large enough and so we can ignore the velocity of sensor $i-1$ in equation (38):

$$
\begin{aligned}
& \dot{x}_{s, i}^{0} \cong-k_{i}\left(x_{s, i}^{0}-x_{s, i-1}\right), \\
& \dot{y}_{s, i}^{0} \cong-k_{i}\left(y_{s, i}^{0}-y_{s, i-1}\right) .
\end{aligned}
$$

From the fact

$$
\begin{aligned}
x_{s, i}^{0}-x_{s, i-1} & =d_{0, i} \cos \left(\beta_{0, i}+\varphi_{i}\right)-d_{i} \cos \left(\beta_{i}+\varphi_{i}\right), \\
y_{s, i}^{0}-y_{s, i-1} & =d_{0, i} \sin \left(\beta_{0, i}+\varphi_{i}\right)-d_{i} \sin \left(\beta_{i}+\varphi_{i}\right),
\end{aligned}
$$

and combining equations (37) and (39), the formation control (36) for sensor $i$ is given by

$$
\begin{aligned}
v_{\mathrm{s}, \mathrm{i}} & =\frac{k_{i}}{\cos \beta_{0, i}}\left(d_{i} \cos \left(\beta_{i}-\beta_{0, i}\right)-d_{0, i}\right), \\
\mathrm{w}_{\mathrm{i}} & =\frac{k_{i}}{d_{0, i} \cos \beta_{0, i}}\left(d_{i} \sin \beta_{i}-d_{0, i} \sin \beta_{0, i}\right)-\dot{\beta}_{0, i} .
\end{aligned}
$$

Then the result about the equilateral-triangle sensor deployment can be stated as follows:

Proposition 14 Under assumption that the velocity of sensor $i-1$ is zero, for sensor $i$ with the modified unicycle model (34) with control (40) and any initial values $\beta_{i}(0), \beta_{0, i}(0)(\in[-\pi,-\pi / 2) \bigcup(-\pi / 2, \pi / 2) \bigcup(\pi / 2, \pi])$, for all positive constants $\epsilon_{1}, \epsilon_{2}$, there exist positive numbers $k_{i}, \varrho$ and $T_{0}$ such that for every $t>T_{0}$

$$
\left|d_{i}-d_{i, 0}\right|<\epsilon_{1},\left|\beta_{i}-\beta_{i, 0}\right|<\epsilon_{2} .
$$

Proof: Consider system (39). Let $e_{x}^{i}=x_{s, i}^{0}-x_{s, i-1}, e_{y}^{i}=$ $y_{s, i}^{0}-y_{s, i-1}$, then we have

$$
\begin{aligned}
\dot{e}_{x}^{i} & =-k_{i} e_{x}^{i}-\dot{x}_{s, i-1}, \\
\dot{e}_{y}^{i} & =-k_{i} e_{y}^{i}-\dot{y}_{s, i-1} .
\end{aligned}
$$

Under Assumption 13 we have from (41) that

$$
\begin{aligned}
& \left|e_{x}^{i}(t)\right| \leq e^{-k_{i} t}\left|e_{x}^{i}(0)\right|+\max \left(k_{v}, k_{s}\right) / k_{i} \\
& \left|e_{y}^{i}(t)\right| \leq e^{-k_{i} t}\left|e_{y}^{i}(0)\right|+\max \left(k_{v}, k_{s}\right) / k_{i}
\end{aligned}
$$

The conclusions follow since

$$
d_{i, 0}-\sqrt{e_{x}^{i}(t)^{2}+e_{y}^{i}(t)^{2}} \leq d_{i} \leq d_{i, 0}+\sqrt{e_{x}^{i}(t)^{2}+e_{y}^{i}(t)^{2}}
$$

and

$$
\tan \left(\phi_{i}+\beta_{i}\right)=\frac{d_{0} \sin \left(\phi_{i}+\beta_{i, 0}\right)-e_{y}^{i}(t)}{d_{0} \cos \left(\phi_{i}+\beta_{i, 0}\right)-e_{x}^{i}(t)} .
$$

Remark 15 When the sensors achieve the desired formation, Assumptions 6 and 9 will be guaranteed.

\section{Conclusion}

In this paper, we designed a nonlinear observer-based filter to track a single second order linear Gaussian target by using measurements from $N$ scalar range sensors and analyzed the the stability of the proposed filter in the sense of mean square. In order to ensure the feasibility of the tracking filter, an equilateral-triangle sensor deployment was proposed for the convergence of the filter by neighbor-based formation control. A very challenging topic for future research is the tracking of multiple targets using mobile sensors. With issues such as data association to overcome, it is far from trivial to extend the results for a single target to such cases.

\section{References}

Agre J, \& Clare L.(2000). An integrated architecture for cooperative sensing networks. Computer, 33(5), 106108.

Kumar S., Zhao F., \& Shepherd D. (2002). Collaborative signal and information processing in microsensor networks. IEEE Signal Processing Mag., 19(2),13-14.

Zhao F. , Shin J., \& Reich J. (2002) Information-driven dynamic sensor collaboration for tracking applications. IEEE Signal Processing Magzine, 19(2), 61-72.

Brooks R. , Ramanathan P., \& Sayeed A. M. (2003). Distributed target classification and tracking in sensor networks. Proc. of IEEE, 91(8), 1163-1171.

Martinez S., \& Bullo F. (2006). Optimal sensor placement and motion coordination for target tracking. Automatica, 42(4), 661-668. 
Cheng D., Ghosh B., \& Hu X. (2006). Distributed sensor network for target tracking. in Proc. of MTNS 2006, Kyoto, July.

Julier S. J., \& Uhlmann J. K. (2004). Unscented filtering and nonlinear estimation. Proc. of IEEE,92(3), 401422 .

Sugathadasa S., Martin C., and Dayawansa W. P.(2000). Convergence of extended Kalman filter to locate a moving target inwild life telemetry. in Proc. of the 39th IEEE Conf. on Decision and Control, Sydney, Australia (pp. 2096-2099).

Reif K., Gunther S., Yaz E., \& Unbehauen R. (2000). Stochastic stability of the continuous-time extended Kalman filter. IEE Proc. Contr. Theory and Appl., vol. 147, no. 1, 2000, pp. 45-52.

Baras J. S., Bensoussan A., \& James M. R. (1988). Dynamic observers as asymptotic limits of recursive filters: special cases. SIAM Journal on Applied Mathematics, 48(5), 1147-1158.

Wang X., Xing G., Zhang Y., Lu C., Pless R., \& Gill C. (2003). Integrated coverage and connectivity configuration in wireless sensor networks. in Proceedings of the ACM SenSys, Los Angeles, USA.

Kumar S., Lai T. H., \& Balogh J. (2004). On k-coverage in a mostly sleeping sensor network, In ACM Mobicom, Philadelphia, PA, USA.

Egerstedt M., Hu X., \& Stotsky A. (2001). Control of mobile platforms using a virtual vehicle approach. IEEE Trans. Aut. Control, 46(11), 1777-1782.

Hu J., \& Hu X. (2008). Optimal target trajectory estimation and filtering using networked sensors. Jr. Systems Science \& Complexity, vol. 21, 325-336.

Zakai M. (1967). On the ultimate boundedness of moments associated with solutions of stochastic differential equations. SIAM J. Control, 5(4),588-593. 